\title{
Operational experience of the ATLAS Pixel Detector
}

Michal Marcisovsky

Institute of Physics, Academy of Sciences of the Czech Republic

on behalf of the ATLAS Collaboration

\section{Introduction}

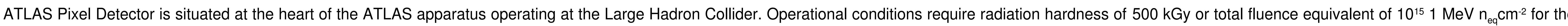
innermost layer. Among the major challenges is fast signal readout and hit identification, detector should be able to distinguish between hits in following bunch crossings with time interval $25 \mathrm{~ns}$. Since the commissioning in 2008

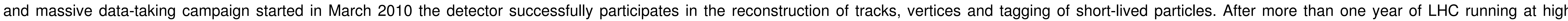
luminosity, more than $1 \mathrm{fb}^{-1}$ of collisions was recorded and significant operational experience was acquired.

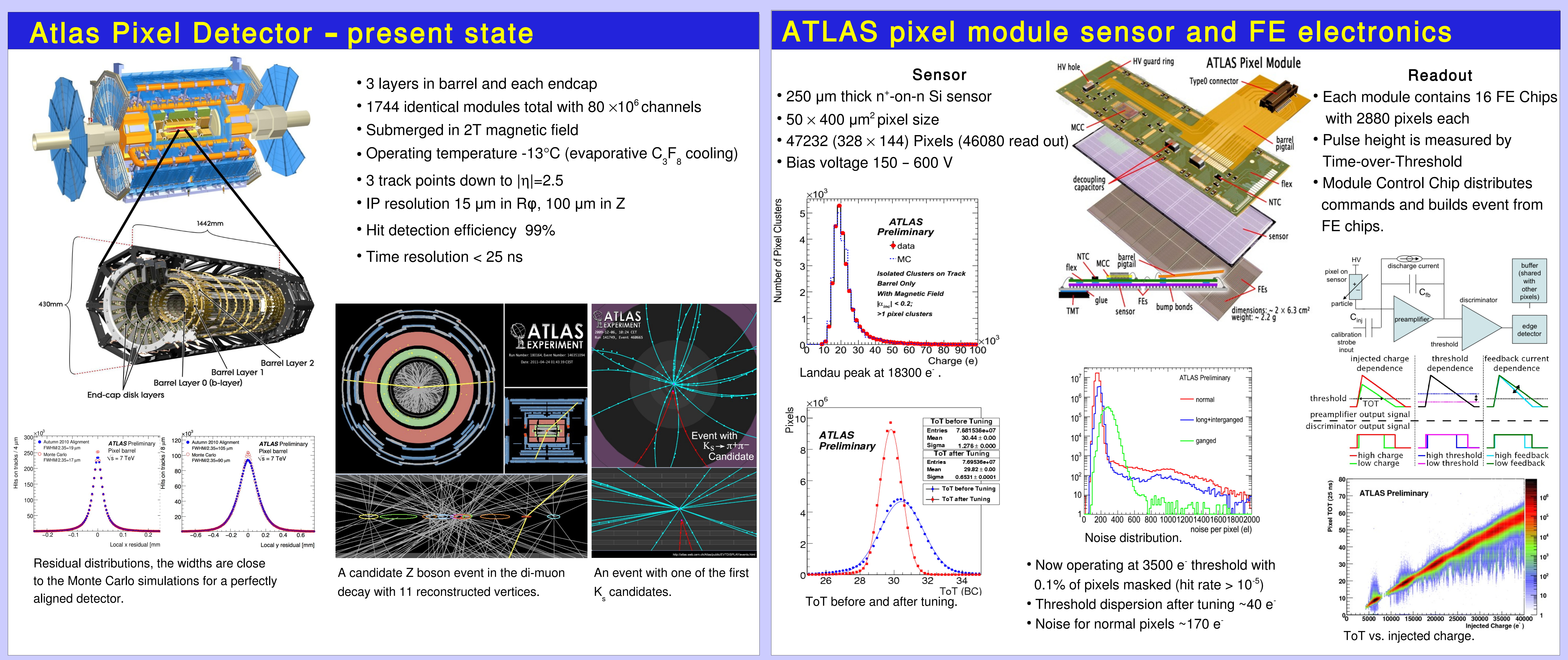

\section{Pixel measurements}

Lorentz angle measurements of $\mathrm{E} \times \mathrm{B}$ drift.

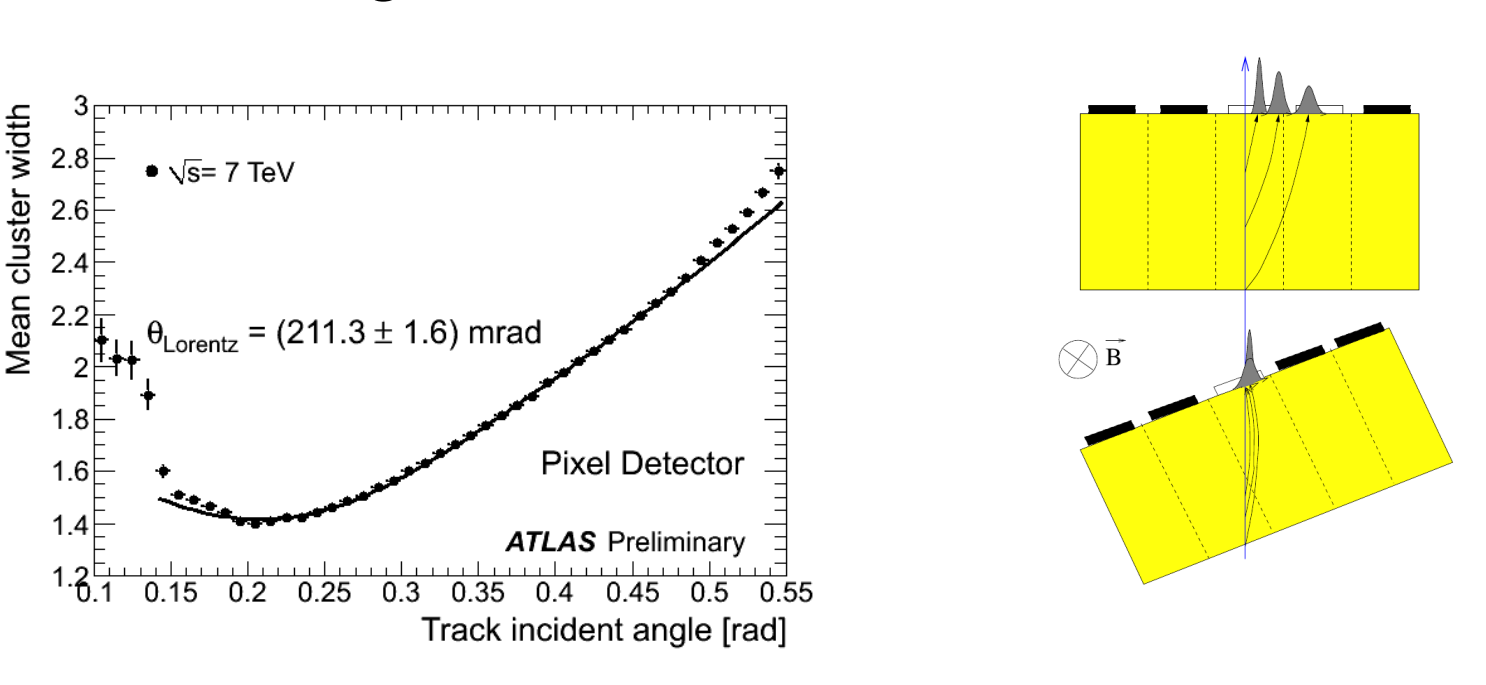

Minimal cluster size is reached for particles coming at a Lorentz angle w.r.t the sensor plane. Measured value of $211.3 \pm 1.5 \mathrm{mrad}$ is close to the expected value of $225 \mathrm{mrad}$

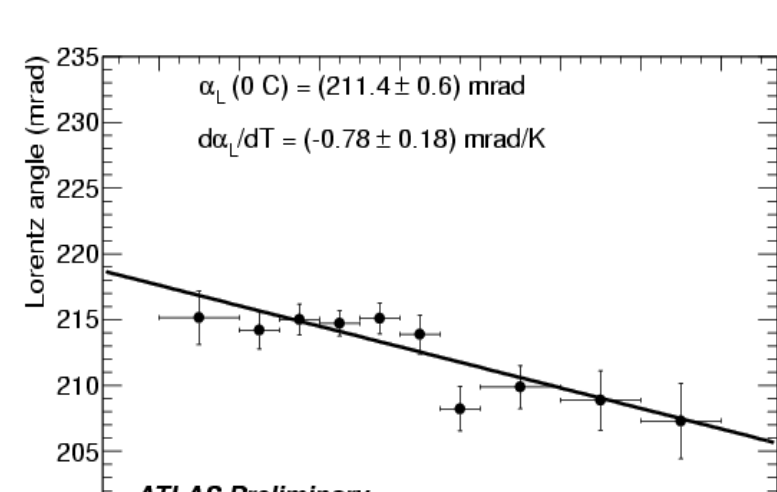

Expected dependence of the Lorentz angle on the mobility measured on modules with different temperature $(-0.78 \pm 0.18) \mathrm{mrad} / \mathrm{K}$, expected: -0.74 $\mathrm{mrad} / \mathrm{K}$

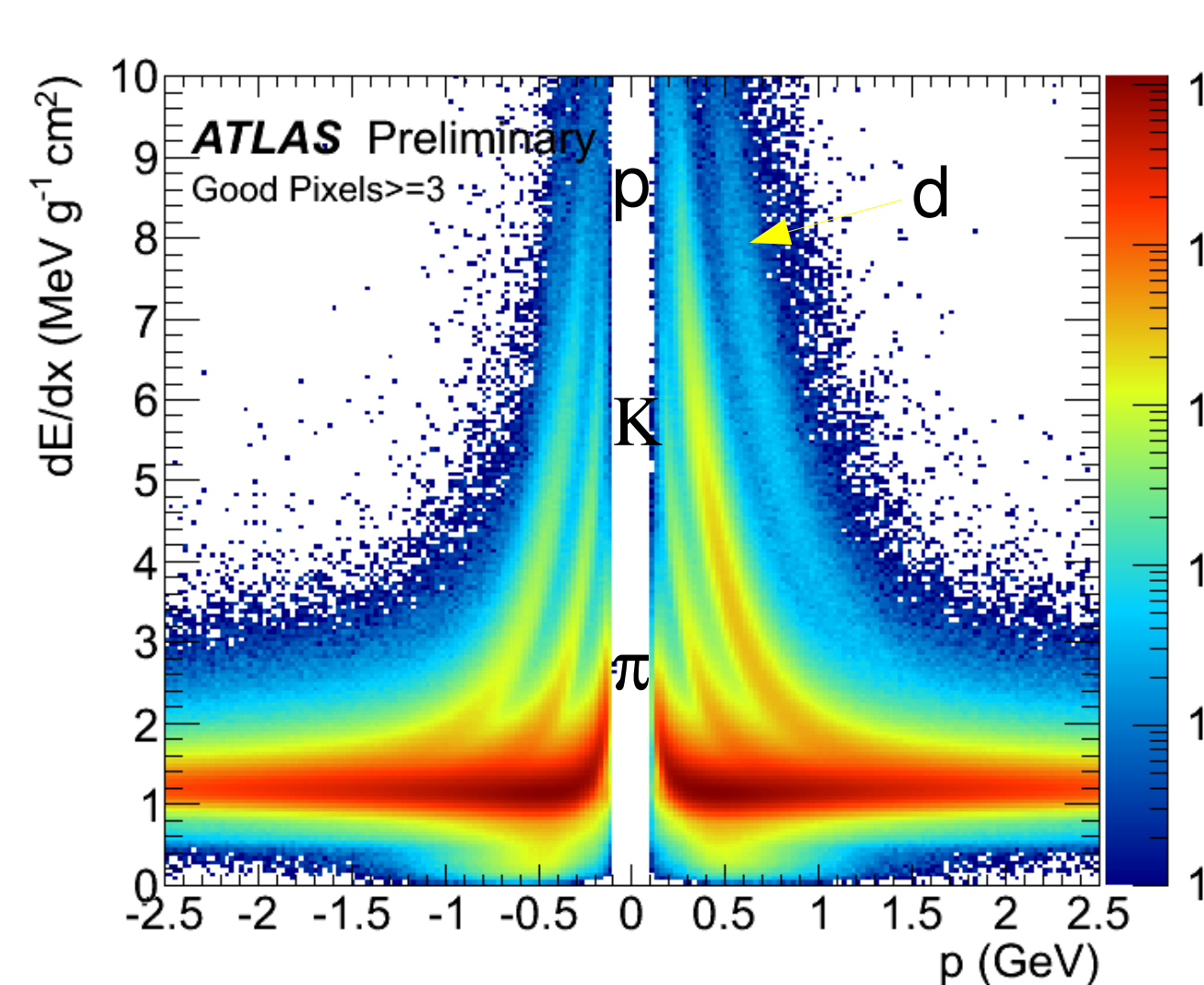

$\mathrm{dE} / \mathrm{dx}$ determined by ToT for different tracks. Pion Kaon, proton and deuteron bands are clearly seen.

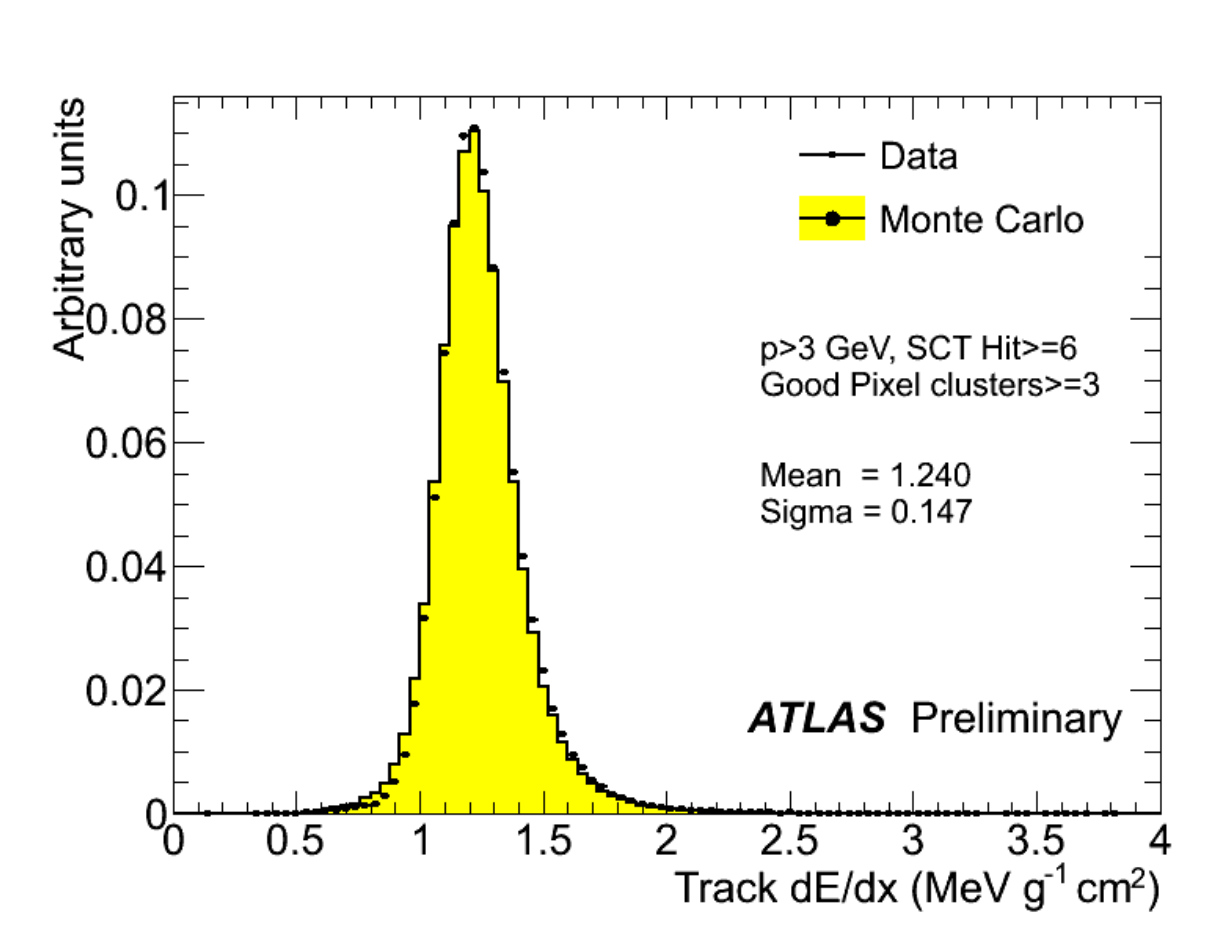

$\mathrm{dE} / \mathrm{dx}$ resolution is $12 \%$.

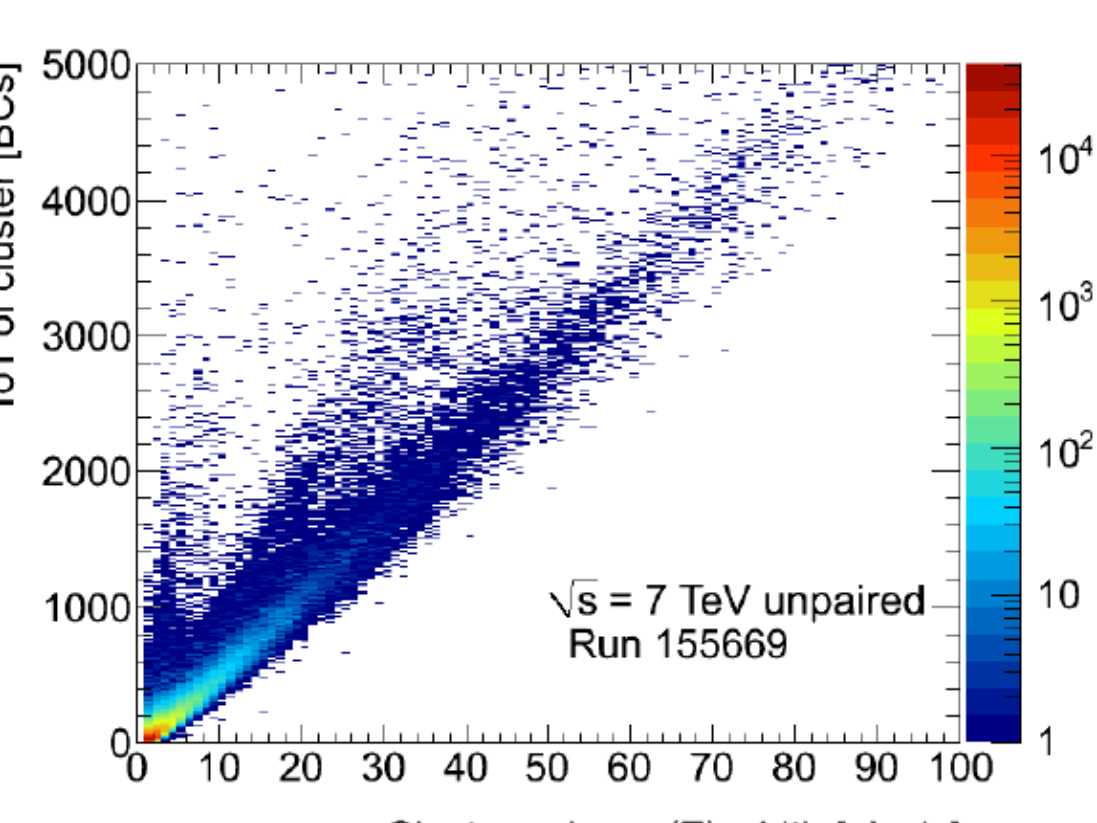

Cluster column (Z) with [pixe

Comparison of cluster colliding bunches.

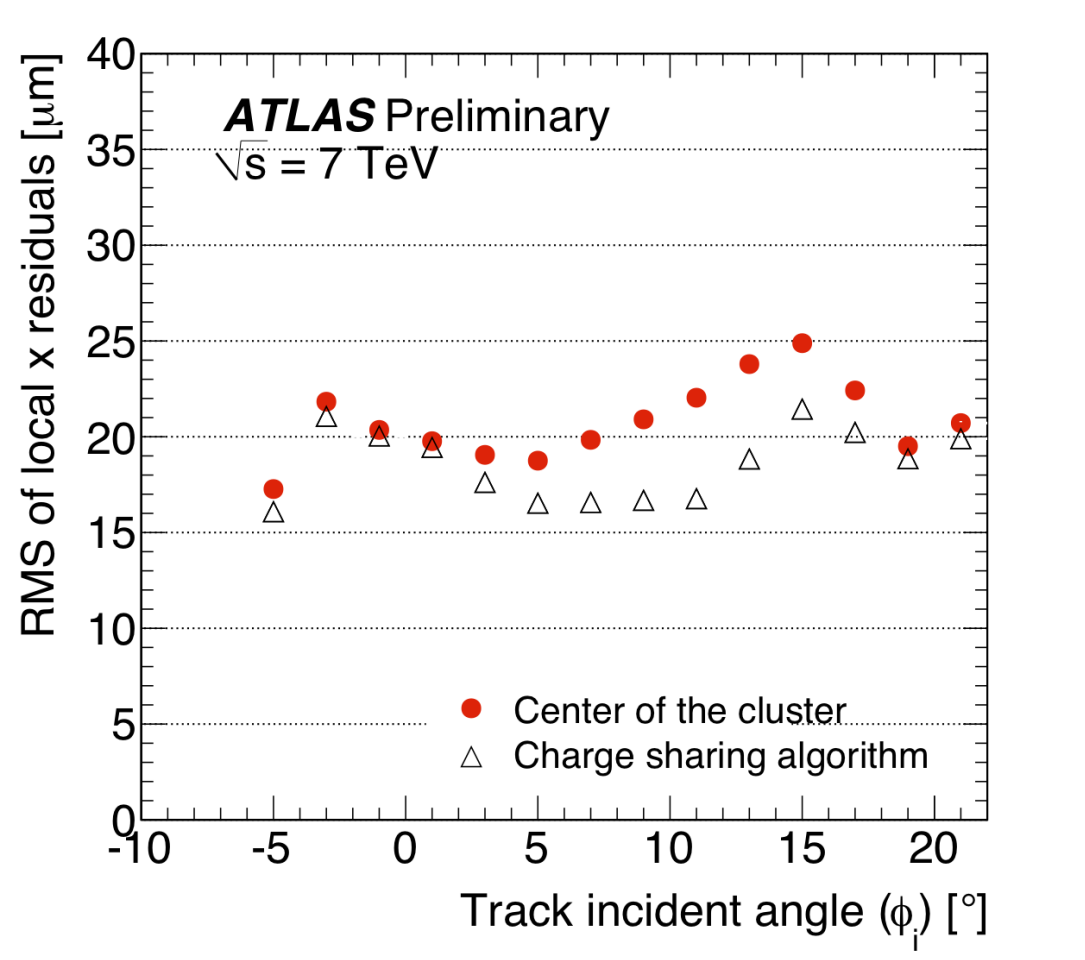

Charge sharing in between pixes on local residuals is remarkable.

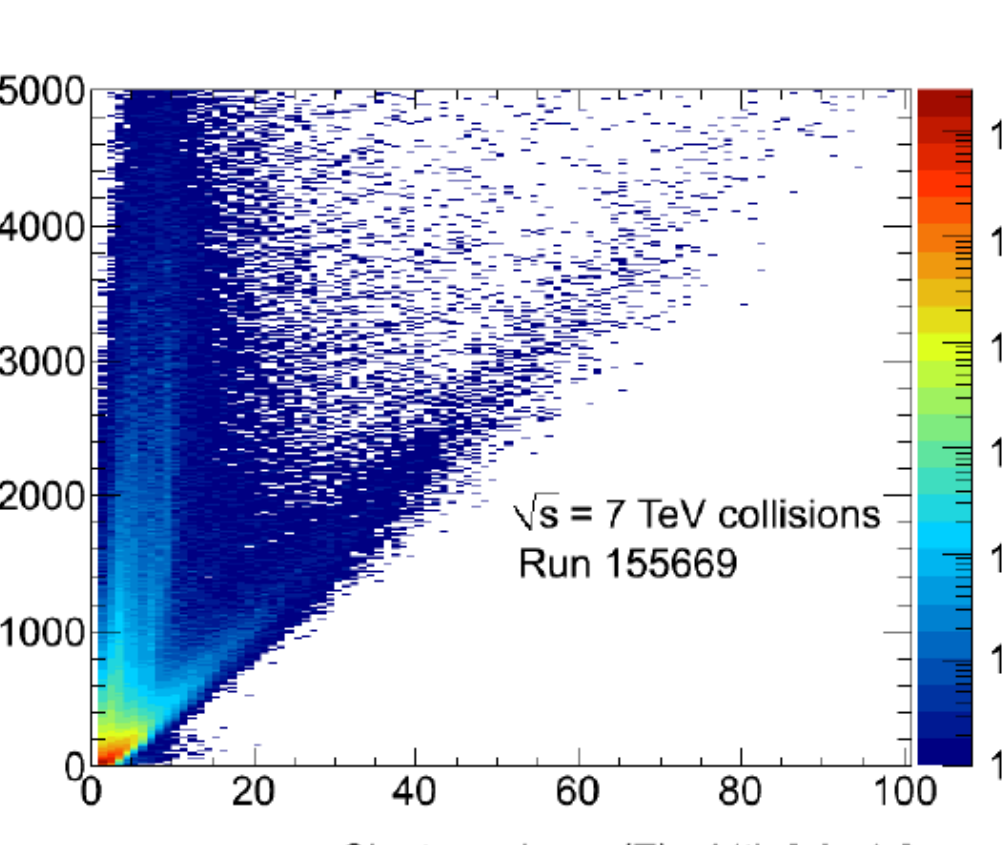

ding vs.

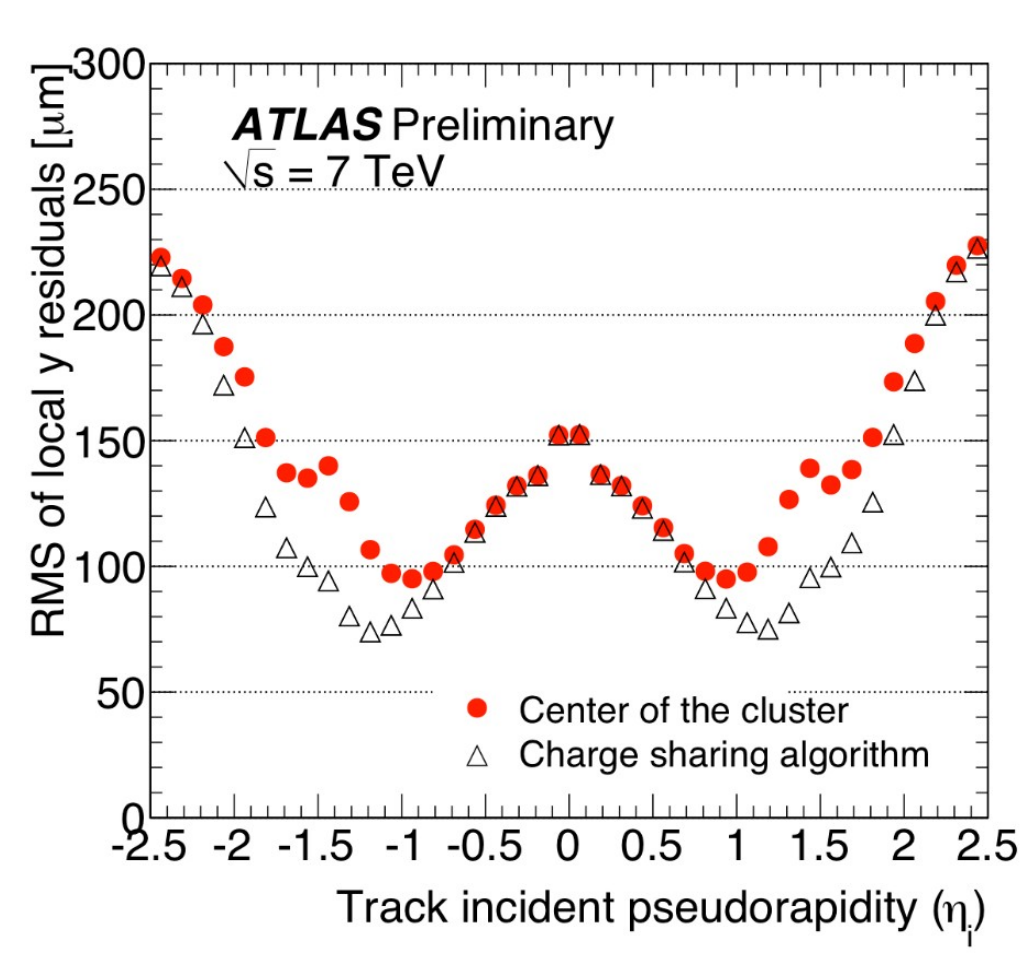

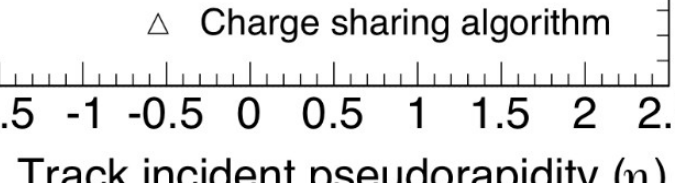

improves the resolution. Impact

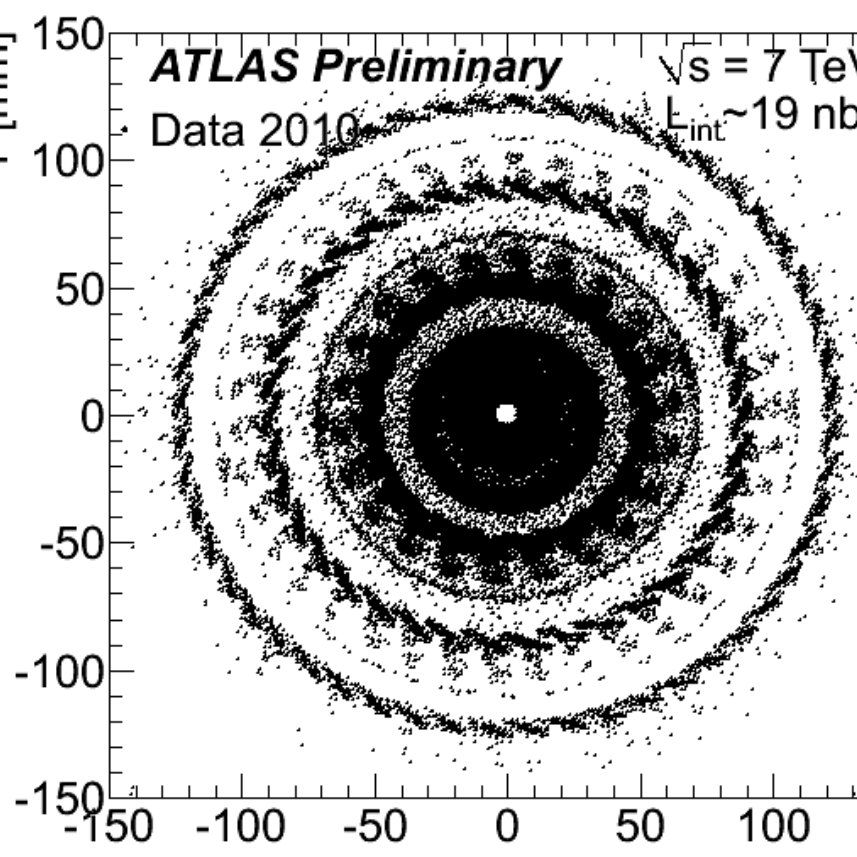

$X$ vs. $Y$ of reconstructed secondary vertices

(excluded vertices from
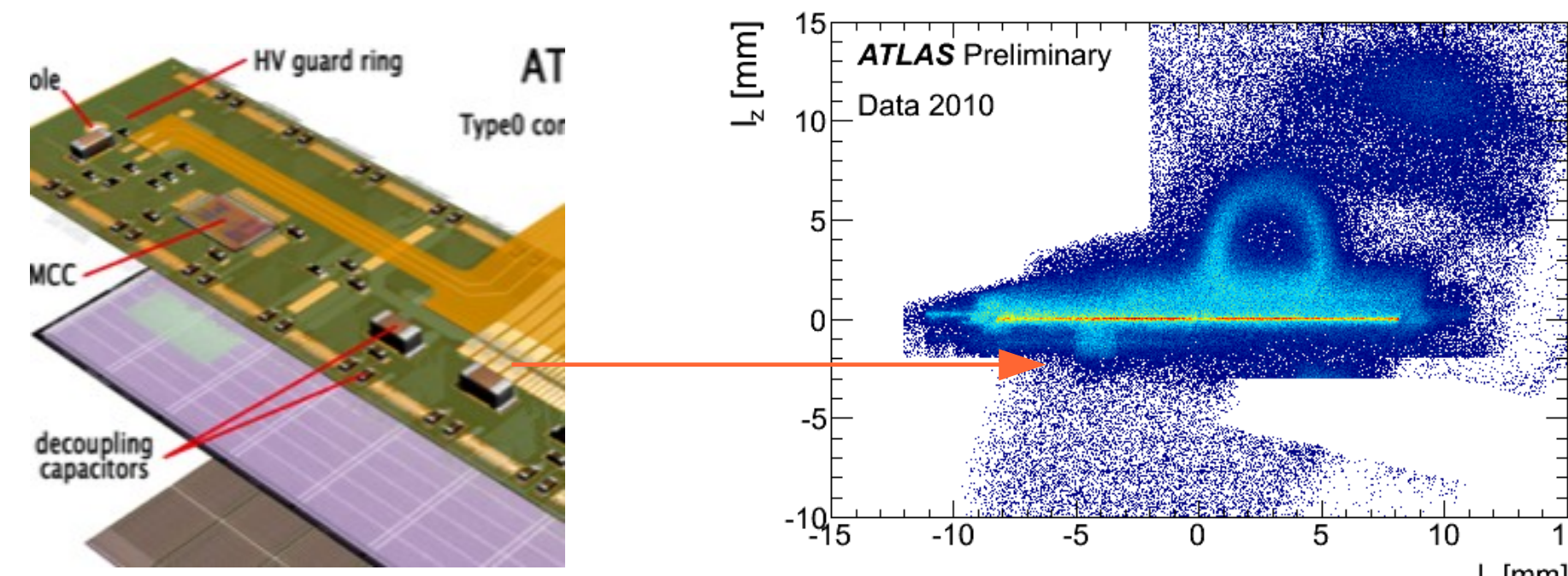

The Al cooling pipe, carbon fiber stave and the sensitive part of the sensor are well visible in both data (lower) and simulation (uper).

\section{Data taking efficiency}
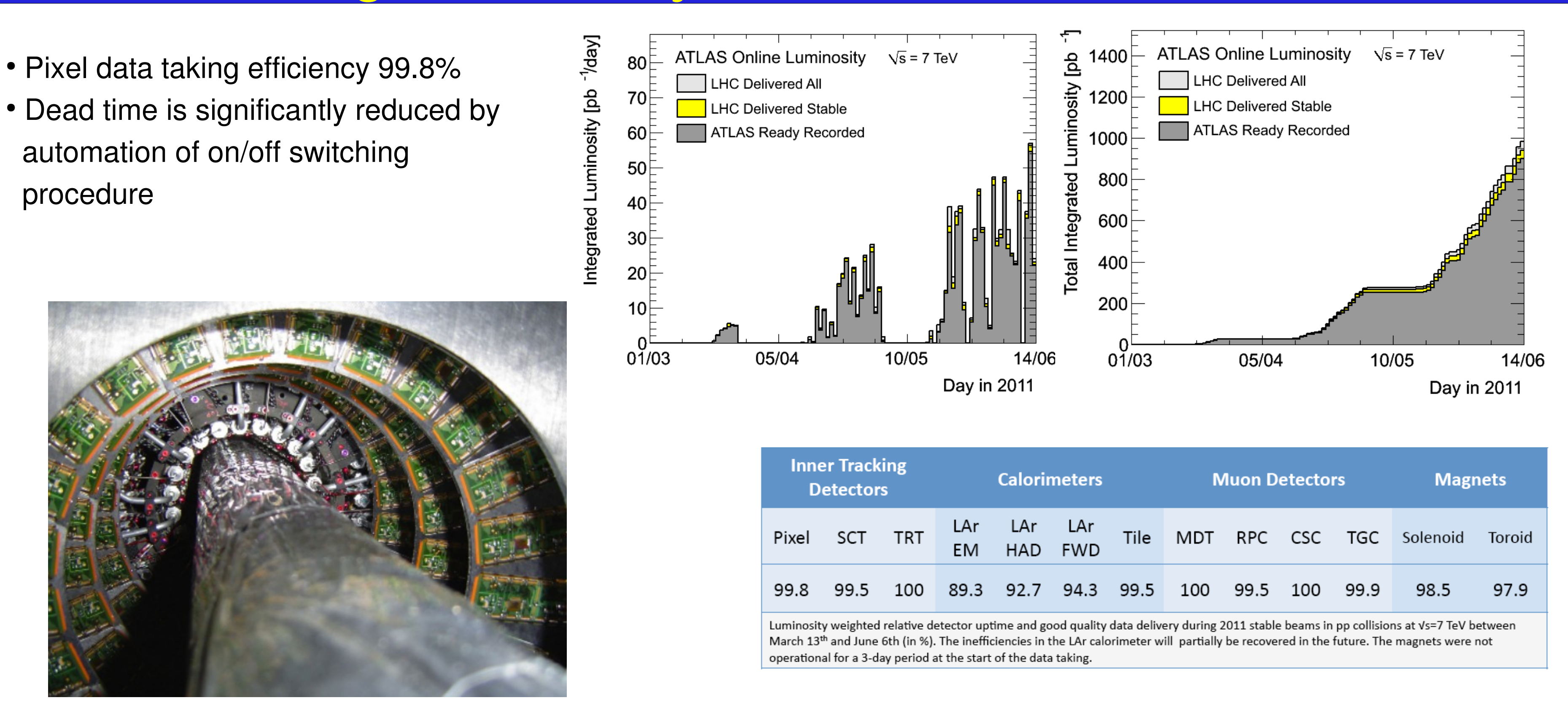

\section{Detection efficiency \\ $96.8 \%$ of the pixels are operational in data taking \\ The fraction of disabled modules increased from \\ Out of 1744 modules: \\ - 55 Modules disabled (3.2\%) \\ - $47 \mathrm{FE}$ chips disabled $(0.16 \%)$ \\ Failures are mostly linked to thermal cycles.}

$\begin{array}{lc}\text { Inactive fraction per layer: } \\ \text { B-layer } & 3.1 \% \\ \text { Layer 1 } & 1.4 \% \\ \text { Layer 2 } & 4.6 \% \\ \text { Endcap A } & 2.8 \% \\ \text { Endcap C } & 2.8 \%\end{array}$

Layer (readout) Occ. Hits/pixel/BC

B-layer $(160 \mathrm{MHz}) \quad 1.5 \times 10^{-4}$

Layer $1(80 \mathrm{MHz}) \quad 0.7 \times 10^{-4}$

Disks $(80 \mathrm{MHz}) \quad 0.55 \times 10^{-4}$

Layer $2(40 \mathrm{MHz}) \quad 0.45 \times 10^{-4}$

Typical module occupancies during data

taking at luminosities $\sim 10^{33} \mathrm{~cm}^{-2} \mathrm{~s}^{-}$

\section{Summary}

Atlas Pixel Detector has been tuned and operates with $99.8 \%$ efficiency, $96.8 \%$ of pixels fully participating in the data taking. Since commissioned, the Pixel Detector demonstrated stability of operational parameters. Requirements on hit detection efficiency, track and vertex reconstruction precision and low noise occupancy rate have been met. Data obtained by the Pixel Detector contributed significantly to the quality of physics results.

Gathered experience with the Pixel Detector demonstrates operational readiness for further data-taking periods and increasing luminosity. 